\section{ORIGINAL RESEARCH}

\section{Calabrese \\ D. Seppi \\ C. Romualdi \\ F. Rinaldi \\ S. Alessio \\ P. Perini \\ P. Gallo}

\title{
Gray Matter Pathology in MS: A 3-Year Longitudinal Study in a Pediatric Population
}

BACKGROUND AND PURPOSE: GM pathology is considered a major determinant of disability in MS, but the comprehension of its origin and progression rate is limited by the uncertainty of dating the biologic disease onset. Thus, we planned a longitudinal study aimed at analyzing and comparing cortical pathology in pediatric and adult MS patients at clinical onset.

MATERIALS AND METHODS: Within 12 months from clinical onset, 35 patients with cMS and 57 with aMS were included in a longitudinal study. At TO, GMf and CL number and volume were analyzed. Percentages of $\Delta$-GMf and number of new CLs were assessed every year for 3 years (T1-T3). Twenty-eight age- and sex-matched NCs constituted the reference population.

RESULTS: At T0, GMf did not differ between cMS and NC $(P=.18)$, while it was lower in patients with aMS compared with both NCs $(P<.001)$ and patients with cMS $(P<.001)$. The number of patients with CLs, as well as CL number and volume, were higher in patients with aMS than in those with cMS $(P<.001)$. At T3, $\Delta$-GMf was higher in both patients with cMS $(1.6 \% \pm 0.5 \%$; range $0.7 \%-3.4 \% ; P<$ $.001)$ and aMS $(1.6 \% \pm 0.6 \%$; range $0.6 \%-3.4 \% ; P<.001)$ compared with NCs $(0.7 \% \pm 0.2 \%$; range $0.4 \%-1.1 \%)$, whereas no difference was observed between patients with CMS and aMS ( $P=.93)$. $\Delta$-GMf significantly correlated with increased CL volume (cMS: $r=0.46$; aMS: $r=0.48$ ) and with the appearance of new CLs (cMS: $r=0.51$; aMS: $r=0.49$ ).

CONCLUSIONS: Our findings suggest that focal (CLs) and diffuse (atrophy) GM damage are strictly associated with the biologic onset of MS, and proceed linearly and partly independently of WM pathology.

ABBREVIATIONS: aMS = adult-onset multiple sclerosis; $\mathrm{CL}=$ cortical lesion; $\mathrm{cMS}=$ childhoodonset multiple sclerosis; EDSS = Expanded Disability Status Scale; GM = gray matter; GMf = gray matter fraction; $\Delta-\mathrm{GMf}=$ delta gray matter fraction; $\Delta-\mathrm{GMf}_{-} 1=$ delta gray matter fraction at $\mathrm{T} 1$; $\Delta$-GMf_2 = delta gray matter fraction at T2; $\Delta-\mathrm{GMf}_{3} 3=$ delta gray matter fraction at $\mathrm{T} 3 ; \mathrm{NC}=$ healthy control; $\mathrm{TO}=$ baseline; $\mathrm{T} 2 \mathrm{WMLV}=\mathrm{T} 2$ white matter lesion volume

$\mathbf{M}$ ultiple sclerosis, though generally considered an inflammatory disease of the WM of the central nervous system, ${ }^{1}$ is characterized by a relevant atrophy of cortical and deep GM.,3 Recently, the view of GM atrophy as a late phenomenon due to retrograde degeneration of damaged axons within WM lesions ${ }^{4,5}$ has been revised on the basis of pathologic ${ }^{6}$ and MR imaging data, ${ }^{7-10}$ suggesting that the neuronal loss in MS occurs early and evolves partly independently of WM inflammation.

However, because it is often impossible to date the biologic or subclinical onset of MS that, in adult patients, may precede its clinical onset by some months to several years, the assessment of how early GM atrophy begins and its evolution, as well as its relationship with WM inflammation, is very difficult. Thus, we thought that further information on the initiation and evolution of cortical damage in MS could be obtained by comparing cMS and aMS. Our working hypothesis was based on the assumption that the interval of time between the biologic and the clinical onset of the disease, though unpredict-

Received July 28, 2011; accepted after revision November 7.

From The Multiple Sclerosis Centre of Veneto Region-First Neurology Clinic (M.C., D.S., F.R., S.A., P.P., P.G.), Department of Neurosciences, University Hospital of Padua, Italy; and Department of Biology (C.R.), University of Padua, Padua, Italy.

Dr. M. Calabrese had full access to all of the data in the study and takes responsibility for the integrity of the data and the accuracy of the data analysis. Dr. Calabrese wrote the first draft of the manuscript and no honorarium, grant, or other form of payment was given to anyone to produce the manuscript.

Please address correspondence to Dr. Massimiliano Calabrese, Multiple Sclerosis Centre of Veneto Region, First Neurology Clinic, Department of Neurosciences, University Hospital of Padua, Via Giustiniani 5, 35128 Padua, Italy; e-mail: calabresem@hotmail.it

http://dx.doi.org/10.3174/ajnr.A3011 able, should probably be shorter in cMS than in aMS. Indeed, MS is extremely rare before the age of 10 years, while its prevalence dramatically increases after 12 years, ${ }^{11-13}$ thus making it unlikely that a teenager has a long preclinical disease phase. Therefore, 2 cohorts of MS patients (pediatric and adult) were included, at clinical onset, in a 3-year longitudinal study aimed at evaluating the presence and the rate of progression of focal and diffuse GM pathology (cortical lesions and atrophy).

\section{Materials and Methods}

\section{Subjects}

Between January 2005 and December 2007, 35 cMS (25 females, 10 males; F:M = 2.5) and 57 patients with aMS (40 women, 17 men; $\mathrm{F}: \mathrm{M}=2.3$ ) were enrolled into a 3-year clinical and MR imaging longitudinal prospective study. All had the relapsing-remitting form of the disease and were included in the study within 12 months from clinical onset (Table 1). Twenty-eight patients with cMS and 42 with aMS had a dissemination in space of the lesions according to Barkhof criteria, ${ }^{14}$ while 7 patients with cMS and 15 with aMS had a dissemination in space of the lesions according to McDonald/Polman criteria (ie, at least $2 \mathrm{WM}$ lesions and presence of intratechal immunoglobin G synthesis). ${ }^{15}$ At study entry (T0), and after 12 (T1; mean $=12 \pm 1$, range 11-14), 24 (T2; mean $=22 \pm 1$, range $20-23$ ), and 36 months (T3; mean $=36 \pm 2$, range 33-39), all patients were clinically evaluated by the EDSS $^{16}$ and underwent MR imaging examination.

Twenty-eight patients with cMS and 53 with aMS were already treated with immunomodulatory agents at study entry (mean time since drug initiation $=8 \pm 6$ months, range $4-18$ ), while the remain- 


\begin{tabular}{|c|c|c|c|}
\hline & $\mathrm{NC}(n=28)$ & cMS $(n=35)$ & $\mathrm{aMS}(n=57)$ \\
\hline Age (years) & $13.3 \pm 2.8(10-16)$ & $13.2 \pm 2.0(8-16)$ & $37.4 \pm 7.2(18-55)^{a, b}$ \\
\hline Age at onset (years) & n.a. & $12.5 \pm 1.8(7-15)$ & $36.9 \pm 6.9(18-54)^{b}$ \\
\hline Patients with BOlgG & n.a & $30(85.7 \%)$ & $51(89.5 \%)$ \\
\hline EDSS-baseline & n.a. & $1.4 \pm 0.8(0-3.0)$ & $3.2 \pm 1.5(1.0-7.0)^{b}$ \\
\hline T2WMLV $\left(\mathrm{cm}^{3}\right)$ & n.a. & $4.0 \pm 5.9(0.2-27.5)$ & $7.4 \pm 9.4(0.3-67.6)^{b}$ \\
\hline Patients with CEL & n.a. & $7(20.0 \%)$ & $12(21.1 \%)$ \\
\hline Number of CEL & n.a. & $1.3 \pm 0.4(1-4)$ & $1.1 \pm 0.3(1-3)$ \\
\hline GMf-baseline \% & $\begin{array}{l}53.9 \pm 2.0 \\
(49.4-57.6)\end{array}$ & $\begin{array}{l}53.0 \pm 2.8 \\
(49.9-56.2)\end{array}$ & $\begin{array}{c}49.9 \pm 3.3 \\
(47.3-53.0)^{\mathrm{a}, \mathrm{b}}\end{array}$ \\
\hline Patients with CLs & n.a. & $12(34.3 \%)$ & $35(61.4 \%)^{b}$ \\
\hline CL number & n.a & $2.1 \pm 1.7(1-12)$ & $3.8 \pm 2.6(1-20)^{b}$ \\
\hline $\mathrm{CL}$ volume $\left(\mathrm{mm}^{3}\right)$ & n.a & $240 \pm 89(0-745)$ & $451 \pm 125(0-1250)^{b}$ \\
\hline
\end{tabular}

Note:-Data are reported as mean \pm standard deviation (range). BOlgG indicates immunoglobin G oligoclonal bands; n.a., not applicable; CEL, contrast-enhancing lesion.

a $P<0.001$ (compared with $\mathrm{NC}$ )

b $P<0.001$ (compared with cMS).

ing 11 patients started on immunomodulatory drugs at study entry. Patients treated with high-dose steroids during the month before MR imaging examination were excluded from the study.

Because a physiologic GM volume reduction occurs in the last phase of adolescence, ${ }^{17}$ a group of 28 healthy adolescents (NC; 20 females, 8 males; F:M = 2.5) were included in the study. This group was age-matched $(P=.949)$ and sex-matched $(P=.979)$ to the cMS group and was composed of adolescents who had a neurologic evaluation for symptoms suggestive of a neurologic disease (including inflammatory disorders of the central nervous system) and who underwent neurologic and MR imaging follow-up. These patients had a final diagnosis of headache or psychological disorder. The local ethics committee approved the study and informed consent was obtained from the patients.

\section{Image Acquisition}

All images were acquired using a $1.5 \mathrm{~T}$ scanner. The following images were obtained from each subject: 1) 3D fast-field echo sequence: 120 contiguous axial sections with the off-center positioned on 0 , TR $=25$ msec, $\mathrm{TE}=4.6 \mathrm{msec}$, flip angle $=30^{\circ}$, section thickness $=1.2 \mathrm{~mm}$, matrix $=256 \times 256$, acquisition time $=7$ minutes 32 seconds; 2) FLAIR: $\mathrm{TR}=10,000 \mathrm{msec}, \mathrm{TE}=120 \mathrm{msec}, \mathrm{TI}=2500 \mathrm{msec}$, echo-train length $=$ 23,50 contiguous axial sections with thickness $=3.0 \mathrm{~mm}$, matrix size $=$ $172 \times 288$, FOV $=250 \times 200 \mathrm{~mm}$, acquisition time $=5$ minutes 2 seconds; 3) double inversion recovery: $\mathrm{TR}=15,631 \mathrm{msec}, \mathrm{TE}=25 \mathrm{msec}$, $\mathrm{TI}=3400 \mathrm{msec}$, delay $=325 \mathrm{msec}$, echo-train length $=17,50$ contiguous axial sections with thickness $=3 \mathrm{~mm}$, matrix size $=130 \times 256$, $\mathrm{FOV}=250 \times 200 \mathrm{~mm}$, acquisition time $=5$ minutes 41 seconds; 4 ) postcontrast T1-weighted spin-echo: $\mathrm{TR}=618 \mathrm{msec}, \mathrm{TE}=10 \mathrm{msec}, 20$ sections with thickness $=5.5 \mathrm{~mm}$, flip angle $90^{\circ}$, matrix size $=224 \times 256$, FOV $=230 \times 230 \mathrm{~mm}$. This sequence was acquired 5 minutes after gadolinium-EDTA $(0.1 \mathrm{mmol} / \mathrm{kg})$ intravenous administration.

\section{Image Analysis}

White and Gray Matter Lesion Number. All images were assessed by consensus by 2 experienced observers (M.C. and D.S.), who were blinded to patient identity. At T0, the number of CLs was assessed on double inversion recovery images following the recent recommendations for CL scoring in patients with MS, ${ }^{18}$ with a count and classification interobserver variability of $2 \%$. The lesions were recognized for their high signal intensity and were distinguishable as lesions confined within the cortical ribbon and not involving the underlying subcortical white matter (Fig 1A-F). At T0, the number of contrast-enhancing lesions was also scored, whereas at T1, T2, and T3, only the number of new CLs and new T2 WM lesions were evaluated.
White and Gray Matter Lesion Volume. At baseline, the CL volume was calculated as previously described. ${ }^{19}$ Briefly, a region of interest was manually drawn around each identified CL, which was then segmented using a thresholding technique based on the Fuzzy Cmeans algorithm, part of the Medical Images Processing, Analysis, and Visualization software (http://mipav.cit.nih.gov). This semiautomated procedure allows the calculation of individual CL volumes and the exclusion of possible double inversion recovery-related hyperintense artifacts. The same procedure was applied to FLAIR images at T0, to identify and segment WM lesions, thus obtaining a T2WMLV.

Gray Matter Fraction Calculation. The 3D-fast-field echo images (at T1, T2, and T3) were firstly coregistered with T0 image using FMRIB Linear Image Registration Tool (part of the FMRIB software library). Therefore, the tissue probability maps for the WM, GM, and CSF were computed for each subject and each time point on volumetric images using SPM8 segmentation (Wellcome Department of Imaging Neuroscience, London, United Kingdom) tools with default settings. ${ }^{20}$ Lesion masks were obtained by contouring lesions on the 3D-fast-field echo scans; then, these masks were subtracted from WM, GM, and CSF maps to obtain 4 mutually exclusive tissue masks, with their volumes in milliliters. GMf was calculated as GM volume/total intracranial volume at baseline (GMf-baseline), at T1 (GMf1), at T2 (GMf2), and at the end of the study (GMf-3). The percentage of GM volume change between T0 and T1 ( $\Delta$-GMf_1) was derived as follows: (GMf-baseline - GMf-T1 / GMf-baseline); the $\Delta$-GMf between T2 and T1 ( $\Delta$-GMf_2) was derived as follows: (GMf-1 - GMf-2/GMf-1); the $\Delta$-GMf between T3 and T2 ( $\Delta$-GMf_3) was derived as follows: (GMf-2 GMf-3/GMf-2); finally, $\Delta$-GMf between T3 and T0 (global $\Delta$-GMf) was derived as follows: (GMf-baseline - GMf-3/ GMf-baseline).

\section{Statistical Analysis}

Between-group differences were assessed using an analysis of covariance, including age and treatment status as a covariate and followed by a Tukey test post hoc correction for multiple comparisons. The $t$ test for paired samples was applied to the patient population (cMS + aMS) to evaluate differences between $\Delta$-GMf_1 and $\Delta$-GMf_2, and between $\Delta$-GMf_2 and $\Delta$-GMf_3. Univariate correlations between continuous variables were assessed using the Pearson or Spearman rank correlation coefficients, as appropriate. A stepwise linear regression analysis was performed on the whole MS population to assess the relative contributions of demographic and MR imaging variables in predicting EDSS change and the global $\Delta$-GMf. 


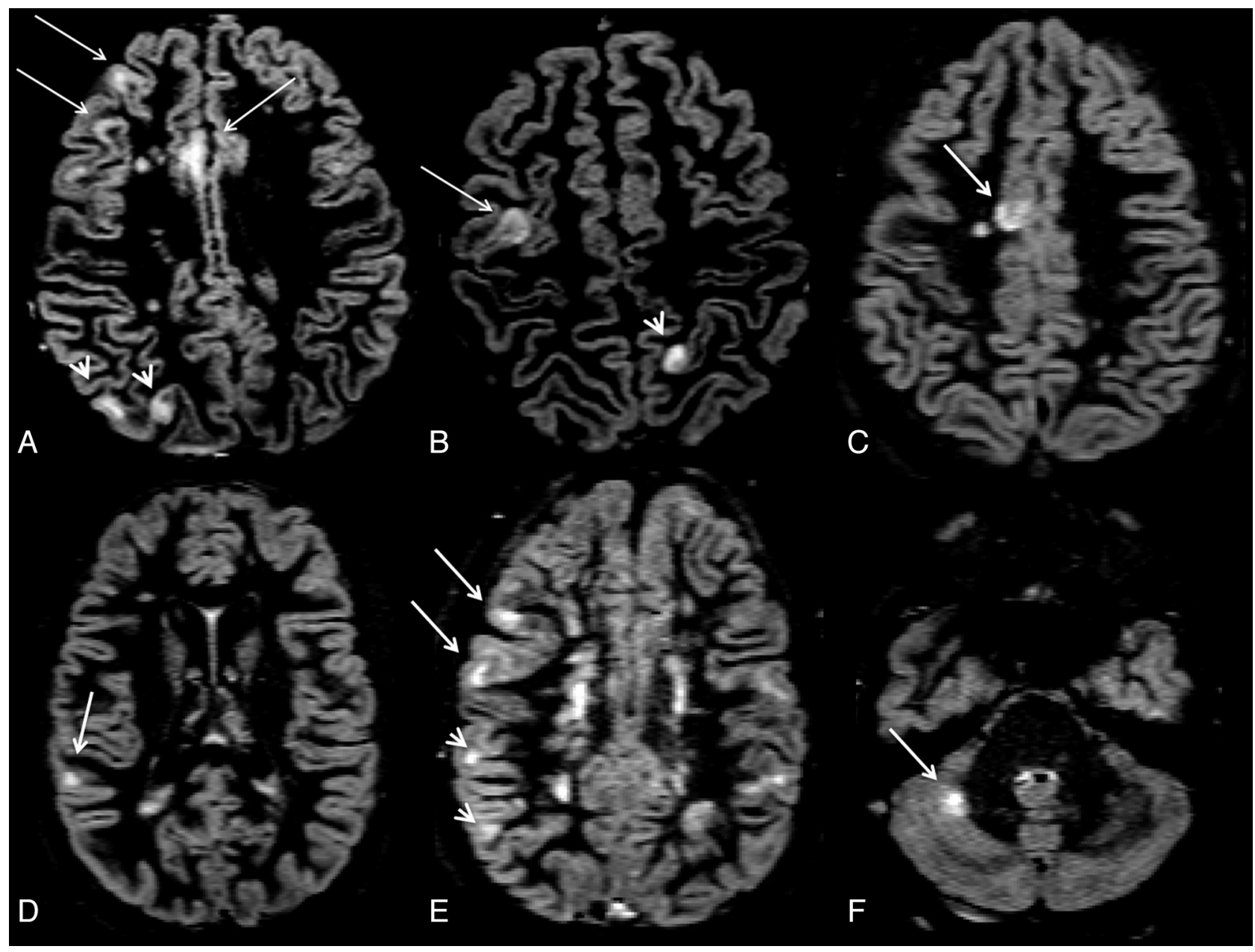

Fig 1. Axial double inversion recovery images at study entry from a 14-year-old adolescent male with relapsing-remitting multiple sclerosis. The EDSS score at study entry was 2.5. Arrows identify pure intracortical lesions, whereas arrowheads identify mixed WM/GM lesions.

\section{Results}

\section{MR Imaging Parameters at Study Entry}

At T0, all MR imaging metrics of cortical pathology were significantly higher in patients with aMS than in those with cMS and NCs; patients with aMS had significantly lower GMf compared with both NCs and patients with $\mathrm{cMS}(P<.001$, even after age correction), while no difference was observed between patients with cMS and NCs in terms of GMf $(P=.18)$.

No difference in the morphology of CLs was observed in patients with cMS compared with those with aMS (Fig 1); however, the number of patients with CLs was higher in those with aMS than in patients with cMS $(P<.001)$; patients with aMS also showed higher CL volume $(P<.001)$, CL number $(P<.001)$, and T2WMLV $(P<.001)$ than patients with cMS, while the number of patients with contrast-enhancing lesion and the contrast-enhancing lesion number did not differ $(P=$ .56 and $P=.41$ ) between the 2 groups (Table 1 ).

\section{MR Imaging Parameters at the End of the Study}

GMf showed a linear reduction in all groups (Fig 2). Indeed, no difference was observed between $\Delta$-GMf_1 and $\Delta$-GMf_2 or between $\Delta$-GMf_2 and $\Delta$-GMf_3 in any of the patient groups (Table 2). However, the progression rate of gray matter atrophy in the aMS and cMS groups was similar and signifi- cantly higher compared with the $\mathrm{NC}$ group. Indeed, at $\mathrm{T} 3$, the global $\Delta$-GMf was significantly higher in both patients with cMS $(1.6 \% \pm 0.5 \%$, range $0.7 \%-3.4 \%, P<.001)$ and aMS $(1.6 \% \pm$ $0.6 \%$, range $0.6 \%-3.4 \%, P<.001)$ compared with NCs $(0.7 \% \pm$ $0.2 \%$, range $0.4 \%-1.1 \%)$, whereas no difference was observed between patients with cMS and aMS $(P=.93$, Table 3$)$. The number of new CLs and new T2-WM lesions did not significantly differ between the patients with cMS and aMS.

\section{MR Imaging Variables Influencing GMf Progression: Correlation Analysis}

At T0, the correlation between GMf and T2WMLV was mild in both patients with cMS $(r=-0.32, P=.01)$ and aMS $(r=$ $-0.29, P=.004)$, while the correlation between GMf and CL volume was much stronger in both groups $(r=-0.68, P<.001$ and $r=-0.54, P<.001$, respectively). No correlation between GMf and contrast-enhancing lesion was observed (data not shown).

At T3, in both cMS and aMS groups, the global $\Delta$-GMf correlated with CL volume $(r=0.46, P<.001$ and $r=0.48$, $P<.001$, respectively) and with new CLs $(r=0.51, P<.001$ and $r=0.49, P<.001$, respectively), while no correlation was observed with T2WMLV and new T2-WM lesions ( $r$ ranged between 0.08 and 0.14 ; $P$ ranged between 0.422 and 0.076 ). 


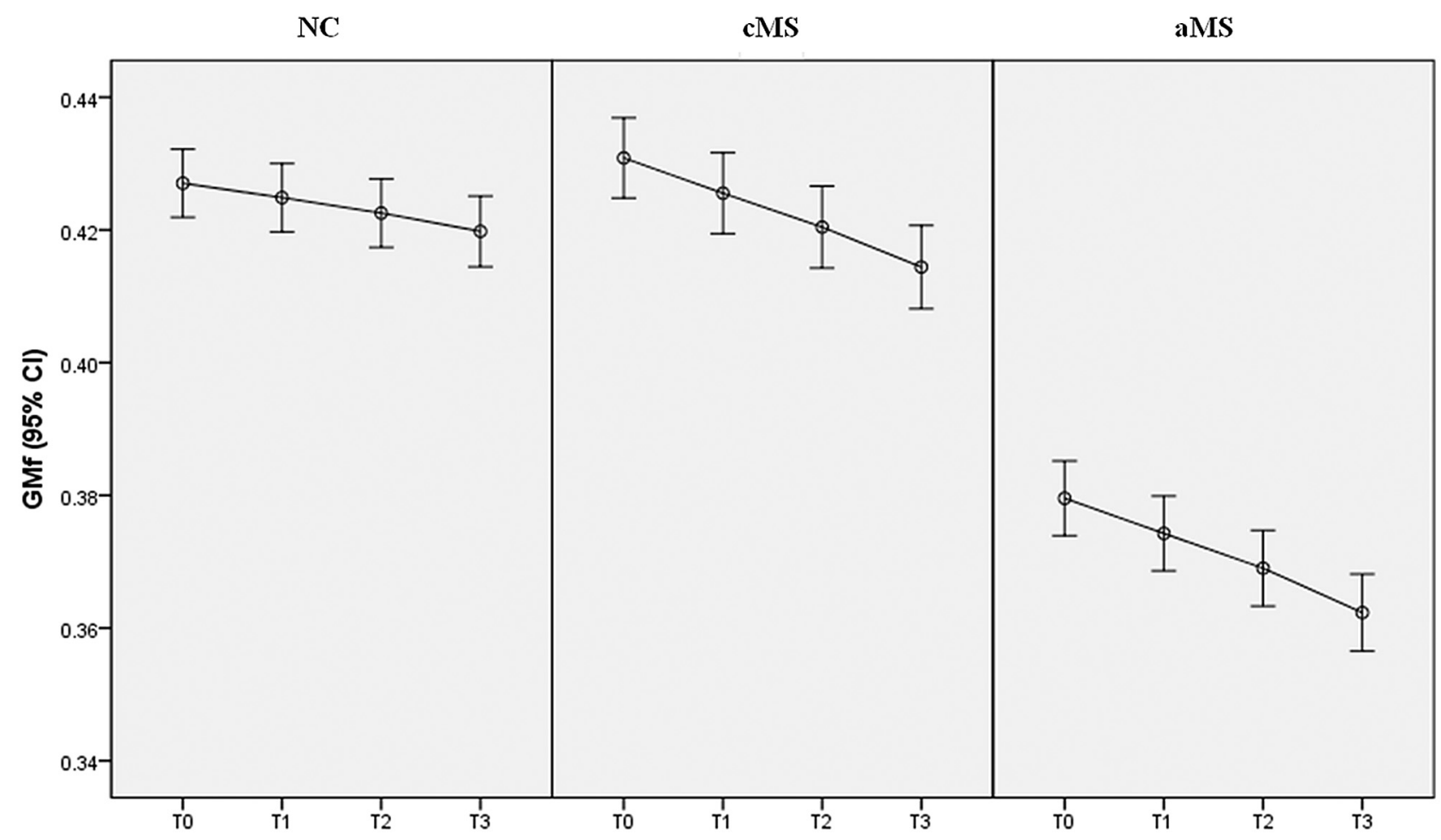

Fig 2. Error bar describing $95 \%$ confidence interval (CI) for mean of GMf at T0, T1, T2, and T3, in NCs, patients with cMS, and patients with aMS.

Table 2: Percentage of gray matter fraction change during follow-up between the $\mathbf{3}$ groups
\begin{tabular}{lcc} 
NC & $0.53 \% 0.20 \%$ & aMS \\
\hline$\Delta$-GMf 1 & $0.22 \% 0.06 \%$ & $0.52 \% 0.27 \%(P=0.749)$ \\
$\Delta$-GMf $2(P$ vs $\Delta$-GMf 1$)$ & $0.23 \% 0.10 \%(P=0.426)$ & $0.60 \% 0.35 \%(P=0.133)$ \\
$\Delta$-GMf $3(P$ vs $\Delta$-GMf 2$)$ & $0.28 \% 0.19 \%(P=0.199)$ & $0.51 \% 0.20 \%(P=0.306)$ \\
\hline No & $0.60 \% 0.40 \%(P=0.168)$
\end{tabular}

Note:-Data are reported as mean \pm standard deviation $(P$ value). $\Delta$-GMf_1 $(\%)=$ percentage of GM fraction change between T0 and T1; $\Delta$-GMf_2 (\%) $=$ percentage of GM fraction change between T1 and T2; $\Delta$-GMf_3 $(\%)=$ percentage of GM fraction change between T2 and T3.

Table 3: Clinical and MRI findings of the patients with MS and the NCs after 3 years of follow-up

\begin{tabular}{lccc}
\hline & NC $(n=28)$ & cMS $(n=35)$ & aMS $(n=57)$ \\
\hline EDSS change & n.a. & $0.8 \pm 0.4(0-3.5)$ & $1.0 \pm 0.5(0-3.0)$ \\
Global $\Delta$-GMf \% & $0.2 \pm 0.1(0.1-0.5)$ & $0.5 \pm 0.2(0.2-1.1)^{*}$ & $0.5 \pm 0.2(0.3-1.0)^{*}$ \\
New T2-WM lesions & n.a & $1.8 \pm 1.0(0.1-5.4)$ & $1.9 \pm 1.5(0-8)$ \\
New CLs & n.a. & $1.5 \pm 1.3(0-8)$ & $1.1 \pm 1.5(0-7)$ \\
\hline
\end{tabular}

Note:-Data are reported as mean \pm standard deviation (range). Global $\Delta$-GMf (\%) indicates percentage of GM fraction change between T0 and T3; n.a., not applicable; T2-WM lesions, white matter lesions.

${ }^{*} P<0.001$ (compared with NC)

No correlation was observed between global $\Delta$-GMf and age (cMS: $r=0.11, P=.365$; aMS: $r=0.13, P=.211$ ). Finally, the multivariate analysis disclosed that baseline GMf $(\mathrm{B}=$ $0.231, P=.016)$ and new $\mathrm{CLs}(\mathrm{B}=0.481, P<.001)$ and $\mathrm{CL}$ volume $(\mathrm{B}=0.341, P<.001)$ were independent predictors of $\Delta$-GMf_total.

\section{MR Imaging Variables Influencing Clinical Outcome: Correlation Analysis}

At T0, the EDSS score significantly correlated with GMf ( $r=$ $-0.51, P<.001)$, T2WMLV $(r=0.37, P=.006)$, and CL volume $(r=0.53, P<.001)$, both in the cMS group and in the aMS group. The multivariate analysis revealed significant contributions from $\mathrm{CL}$ volume $(\mathrm{B}=0.523 ; P<.001)$ and $\Delta$-GMf final $(\mathrm{B}=0.482 ; P<.001)$ as independent predictors of EDSS change.

\section{Discussion}

We analyzed the brain cortex in pediatric and adult patients with MS within 1 year from clinical onset and thereafter for 3 years, and observed that both atrophy and cortical lesion volume and number, while significantly greater in the adult population at baseline, had the same rate of progression in the 2 groups. As expected, the progression of cortical atrophy was significantly higher in both MS groups compared with the NC group, thus confirming the hypothesis that GM pathology is a very early phenomenon in MS, even in the pediatric population. ${ }^{7-10,21}$ The lack of a significant difference in GMf between patients with cMS and NCs at study entry is probably due to the short preclinical phase in patients with cMS. Indeed, epidemiological data indicate that MS onset is rare before the age of 10 years, while it increases dramatically after puberty and 
menarche, ${ }^{11-13}$ thus making it unlikely that a teenaged child would have a long preclinical disease phase.

The longitudinal evaluation of the GMf did not show differences in any of the patient groups between $\Delta$-GMf_1 and $\Delta$-GMf_2, or between $\Delta$-GMf_2 and $\Delta$-GMf_3, suggesting that neuronal loss proceeds linearly over time in all groups, independent of patient age, WM lesion load, or WM lesion accumulation. On the contrary, the appearance of new CLs and their increase in volume correlated with GMf progression, and these were found to be independent predictors of GMf change in the multivariate analysis. These findings strengthen previous observations suggesting that local cortical inflammation is related to cortical atrophy in MS. ${ }^{22,23}$ Indeed, patho$\operatorname{logic}^{24}$ and MR imaging studies ${ }^{19,22}$ have observed that CLs, much more frequent than previously supposed, can be detected early during the course of the disease, ${ }^{21}$ and that these accumulate in cortical areas where the degenerative process, and the consequent GM atrophy, is more pronounced. ${ }^{23}$

The correlation observed between the progression of cortical pathology (CL volume and GMf) and EDSS change is particularly relevant from the clinical point of view, because it further suggests that cortical pathology plays a major role in determining the clinical deterioration of patients with MS. ${ }^{25,26}$

We believe that the cMS population, which is probably characterized by the shortest interval of time between the biologic and the clinical onset of the disease within the entire MS population, constitutes a particularly interesting cohort of patients with MS for studies aimed at evaluating the clinical (diagnostic and prognostic) relevance of GM MR imaging parameters. However, we are aware that our preliminary results need to be confirmed by analyzing the evolution of regional GM atrophy in a larger group of patients with cMS.

\section{Conclusions}

The significantly higher progression rate of GM pathology in patients with cMS and aMS compared with NCs suggests that a local pathologic process takes place early in the cortex of patients with MS, even in the pediatric population. The absence of any difference in GMf between patients with cMS and NCs at baseline is probably due to the short disease duration in cMS. The linear evolution of cortical atrophy and the lack of a strong correlation with WM lesion volume, in line with recent literature reports, ${ }^{26-28}$ speak against the "classic" opinion that GM atrophy is mainly due to the retrograde axonal degeneration following axonal transection in WM lesions. Thus, our findings support the growing evidence that MS should be considered a "simultaneous 2-component" disease ${ }^{29}$ in which GM degeneration proceeds largely independent of WM inflammation. Considering the strong relationship between GM pathology and clinical outcome, a major effort should be devoted to identify the immunologic mechanisms responsible for GM changes to develop therapeutic strategies aimed at preventing disability progression in MS.

Disclosures: Massimiliano Calabrese-UNRELATED: Payment for Lectures (including service on speakers bureaus): Sanofi Aventis, Serono Pharma, Biogen Idec, Bayer Schering; Payment for Development of Educational Presentations: Sanofi Aventis, Serono Pharma, Biogen Idec, Bayer Schering; Travel/Accommodations/Meeting Expenses Unrelated to Activities Listed: Sanofi Aventis, Serono Pharma, Biogen Idec, Bayer Schering. Dario Seppi-UNRELATED: Travel/Accommodations/Meeting Expenses Unrelated to Activities Listed: Biogen Idec, Sanofi Aventis, Serono Pharma, Bayer Schering. Francesca Rinaldi-
UNRELATED: Travel/Accommodations/Meeting Expenses Unrelated to Activities Listed: Biogen Idec, Bayer Schering, Novartis, Sanofi Aventis. Paolo Gallo-UNRELATED: Consultancy: Merck Serono, Bayer Schering, Novartis Pharma, Biogen Italy, Sanofi Aventis; Grants/Grants Pending: Merck Serono, Bayer Schering, Novartis Pharma, Biogen Italy, Sanofi Aventis; Payment for Lectures (including service on speakers bureaus): Merck Serono, Bayer Schering, Novartis Pharma, Biogen Italy, Sanofi Aventis.

\section{References}

1. Noseworthy JH, Lucchinetti C, Rodriguez M, et al. Multiple sclerosis. N Engl J Med 2000;343:938-52

2. Chard DT, Miller D. Grey matter pathology in clinically early multiple sclerosis: evidence from magnetic resonance imaging. J Neurol Sci 2009;282:5-11

3. Vercellino M, Masera S, Lorenzatti M, et al. Demyelination, inflammation, and neurodegeneration in multiple sclerosis deep gray matter. J Neuropathol Exp Neurol 2009;68:489-502

4. Simon JH, Kinkel RP, Jacobs L, et al. A Wallerian degeneration pattern in patients at risk for MS. Neurology 2000;54:1155-60

5. Trapp BD, Peterson J, Ransohoff RM, et al. Axonal transection in the lesions of multiple sclerosis. N Engl J Med 1998;338:278-85

6. Pirko I, Lucchinetti CF, Sriram S, et al. Gray matter involvement in multiple sclerosis. Neurology 2007;68:634-42

7. Chard DT, Griffin CM, Rashid W, et al. Progressive grey matter atrophy in clinically early relapsing-remitting multiple sclerosis. Mult Scler 2004;10:387-91

8. Dalton CM, Chard DT, Davies GR, et al. Early development of multiple sclerosis is associated with progressive grey matter atrophy in patients presenting with clinically isolated syndromes. Brain 2004;127:1101-07

9. De Stefano N, Matthews PM, Filippi M, et al. Evidence of early cortical atrophy in MS: relevance to white matter changes and disability. Neurology 2003;60:1157-62

10. Calabrese M, Atzori M, Bernardi V, et al. Cortical atrophy is relevant in multiple sclerosis at clinical onset. J Neurol 2007;254:1212-20

11. Pohl D, Hennemuth I, von Kries R, et al. Paediatric multiple sclerosis and acute disseminated encephalomyelitis in Germany: results of a nationwide survey. Eur J Pediatr 2007; 166:405-12

12. Banwell B, Krupp L, Kennedy J, et al. Clinical features and viral serologies in children with multiple sclerosis: a multinational observational study. Lancet Neurol 2007;6:773-81

13. Atzori M, Battistella PA, Perini P, et al. Clinical and diagnostic aspects of multiple sclerosis and acute monophasic encephalomyelitis in pediatric patients: a single centre prospective study. Mult Scler 2009;3:363-70

14. Barkhof F, Filippi M, Miller DH, et al. Comparison of MRI criteria at first presentation to predict conversion to clinically definite multiple sclerosis. Brain 1997;120:2059-69

15. Polman CH, Reingold SC, Edan G, et al. Diagnostic criteria for multiple sclerosis: 2005 revisions to the "McDonald Criteria." Ann Neurol 2005;58:840-46

16. Kurtzke JF. Rating neurologic impairment in multiple sclerosis: an expanded disability status scale (EDSS). Neurology 1983;33:1444-52

17. Giorgio A, Santelli L, Tomassini V. Age-related changes in grey and white matter structure throughout adulthood. Neuroimage 2010;51:943-51

18. Geurts JJ, Roosendaal SD, Calabrese M, et al. Consensus recommendations for MS cortical lesion scoring using double inversion recovery MRI. Neurology 2011;76:418-24

19. Calabrese M, De Stefano N, Atzori M, et al. Detection of cortical inflammatory lesions by double inversion recovery magnetic resonance imaging in patients with multiple sclerosis. Arch Neurol 2007;64:1416-22

20. Miller DH, Barkhof F, Frank JA, et al. Measurement of atrophy in multiple sclerosis: pathological basis, methodological aspects and clinical relevance. Brain 2002;125:1676-95

21. Calabrese M, Gallo P. Magnetic resonance evidence of cortical onset of multiple sclerosis. Mult Scler 2009;15:933-41

22. Calabrese M, Rocca MA, Atzori M, et al. Cortical lesions in primary progressive multiple sclerosis: a 2-year longitudinal MR study. Neurology 2009;72:1330-36

23. Calabrese M, Battaglini M, Giorgio A, et al. Imaging distribution and frequency of cortical lesions in patients with multiple sclerosis. Neurology 2010;75:1234-40

24. Kidd D, Barkhof F, McConnell R, et al. Cortical lesions in multiple sclerosis. Brain 1999;122:17-26

25. Fisniku LK, Chard DT, Jackson JS, et al. Gray matter atrophy is related to long-term disability in multiple sclerosis. Ann Neurol 2008;64:247-54

26. Calabrese M, Rocca MA, Atzori M, et al. A 3-year magnetic resonance imaging study of cortical lesions in relapse-onset multiple sclerosis. Ann Neurol 2010;67:376-83

27. Kutzelnigg A, Lucchinetti CF, Stadelmann C, et al. Cortical demyelination and diffuse white matter injury in multiple sclerosis. Brain 2005;128:2705-12

28. Bö L, Geurts JJ, VanderValk P, et al. Lack of correlation between cortical demyelination and white matter pathologic changes in multiple sclerosis. Arch Neurol 2007;64:76-80

29. Charil A, Filippi M. Inflammatory demyelination and neurodegeneration in early multiple sclerosis. J Neurol Sci 2007;259:7-15 